\title{
Human Gut Microbiome Metabolism and Autism Spectrum Disorder
}

\author{
Martin Noguera \\ University of Florida \\ United States
}

\begin{abstract}
Human gut microbiome and microbial metabolism correlations with gastrointestinal and extra intestinal disease and/or disorder have been explored heavily in recent years. Interest in correlations with mental health disease and disorder is of interest due to growing mental health issues in the United States and around the world as well. Clinically diagnosed individuals with Autism spectrum disorder exhibit gastrointestinal symptoms such as constipation, dysbiosis, abdominal pain, diarrhea, and increased intestinal permeability. The enteric nervous system can communicate with the central nervous system through neurochemical signals that are stimulated by microbial associated molecular patterns and metabolite production. This so-called gut-brain axis can, therefore, be exploited for novel therapeutics that inhibit atypical neurochemical signaling produced by altered microbial composition and microbial metabolic means.
\end{abstract}

Keywords: Human, Gut, Microbiome, Metabolism, Autism, Disorder

The Importance of Exploring the Human Gut Microbiome and Metabolism for Autism Insight

Correlations between the human gut microbiome (HGM) and neurological disease and disorder have been of growing interest due to increasing mental health issues in the United States as well as other countries. According to the Center for Disease Control, the prevalence of Autism (ASD) has seen an increase at a rate of 1 in 68 children being affected [1]. ASD is a significant area of study concerning HGM as gastrointestinal symptoms are common in ASD individuals. Thus, making it possible that these symptoms may be related to an HGM composition and/or metabolism that is unique to ASD individuals versus neurotypical. Knowing this, it is theorized that therapeutics such as antibiotics, probiotics, prebiotics, and fecal microbial transplant (FMT) may not just ameliorate gastrointestinal symptoms, but also decrease the likelihood of ASD development and/or regression. This review aims to discuss recent findings in HGM, metabolism, its correlation with ASD, and therapeutics that are being considered.

\section{HGM, Metabolism, and ASD}

The gut's nervous system or enteric nervous system can communicate with the central nervous system through neurochemical signals that are microbially promoted. This can be accomplished through either metabolite production or microbial associated molecular patterns. Metabolite production of neurotransmitter compounds such as tryptophan and tyrosine can enter the cardiovascular system, cross the blood-brain barrier, and enter the nervous system [2].This can regulate and/or stimulate different neurological functions. Microbial associated molecular patterns like lip polysaccharide and other metabolites can trigger the immune system and influence neurophysiology [3]. Therefore, it can be assumed that alteration of the gut microbiome composition may affect neurological function and social behavior.

Tryptophan (Trp)is found in common food products such as milk, cheese, bread, and poultry. Although an essential amino acid, it is not produced by animal cells and relies on dietary intake. Trp is also a metabolite involved in hostmicrobiota interaction. Major pathways of Trp metabolism lead to serotonin, kynurenine, and indole derivatives that are controlled to an extent by the gut microbiome. Serotonin (5-HT) is of importance as it is a neurotransmitter that has been described to regulate social behavior and possibly interfere with physiological processes [4]. It has been isolated from Streptococcus, Escherichia, and Enterococcus species [5,6]. Additionally, gut microbial species such as Clostridium sporogenes, Escherichia coli, and lactobacilli play a role in manipulating Trp to different metabolic components, some of which are neurotransmitter like 5-HT, and is suggested that impaired Trp metabolism plays a role in neuropsychiatric traits such as Autism [7,8]. Therefore, if "typical" gut microbiota composition is compromised, so is metabolism, and this can lead to an increased possibility of worsening effects 


\section{HGM and Metabolism, Investigational Studies in ASD}

Kalzuna-Czaplinska et. al 2019, describes studies aiming to compare the metabolic and nutritional status of ASD with controls and investigated autism severity to Trp biomarkers [9-12].One study examined 55 children ages 5-16 with ASD and 44 neurotypical controls with similar age, gender, and geography.

Results showed a significant Trp decrease in the ASD group and might be due to protein intake and/or dysfunction in synthesizing protein into amino acids in the digestive tract [13].Knowing this, we can assume that serotonin levels are also affected and there is a greater possibility of worsening social behavior. Additionally, this may involve impaired Trp metabolism as previously discussed by certain gut microbial species.

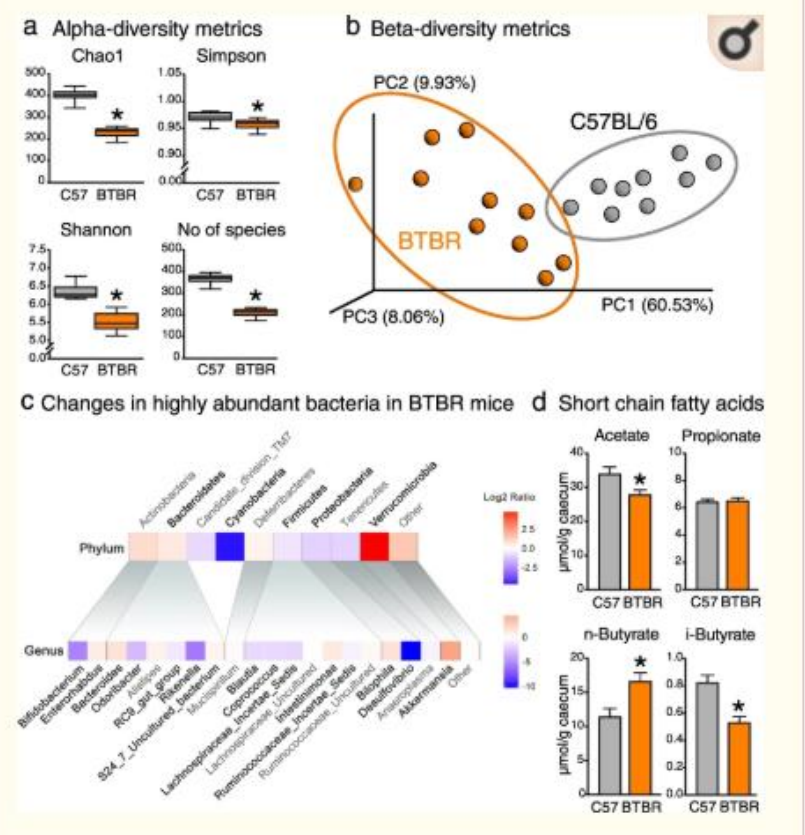

Figure 1.16S sequencing results/data BTBR vs C57BL/6 mice. Golubeva, A., et. al. (2017).

Furthermore, a mouse model of ASD using BTBR (impaired) and C57BL/6 (control) explored interactions between intestinal microbiota, gut physiology, and social behavior. Figure 1 shows data from 16S sequencing of the BTBR and C57BL/6 cecal microbiota. Altered microbiota composition and short-chain fatty acid (SCFA) levels are seen in the BTBR mice as well as lower bacterial diversity $(a, b, d)$. Additionally, a heat map was generated to visualize increases (red) and decreases (blue) in most abundant bacteria in BTBR compared to C57BL/6 mice (c).

Table S2. Relative abundance (\%) of bacterial PHYLA in the caecum of C57BL/6 and BTBR mice. Related to Figure 1

\begin{tabular}{|c|c|c|c|c|c|}
\hline \multirow{2}{*}{ Phylum } & \multicolumn{2}{|c|}{ Median (IQR) } & \multirow{2}{*}{$\begin{array}{c}U_{(17)} \\
\text { value }\end{array}$} & \multirow{2}{*}{ p value } & \multirow{2}{*}{$\begin{array}{c}\text { FDR } \\
\text { adjusted } \\
p \text { value }\end{array}$} \\
\hline & C57BL/6 & BTBR & & & \\
\hline Actinobacteria & $0.154(0.150)$ & $0.317(0.249)$ & 69.000 & 0.053 & 0.089 \\
\hline Bacteroidetes & $37.954(11.813)$ & $56.546(13.264)$ & 88.000 & $<0.0005^{*}$ & $<0.0005^{*}$ \\
\hline Candidate division TM7 & $0.104(0.086)$ & $0.015(0.188)$ & 24.000 & 0.095 & 0.118 \\
\hline Cyanobacteria & $0.038(0.029)$ & $0.000(0.001)$ & 0.000 & $<0.0005^{*}$ & $0.001^{*}$ \\
\hline Deferribacteres & $0.963(0.515)$ & $0.192(1.650)$ & 29.000 & 0.211 & 0.234 \\
\hline Firmicutes & $59.238(11.340)$ & $40.151(13.061)$ & 2.000 & $<0.0005^{*}$ & $<0.0005^{*}$ \\
\hline Proteobacteria & $1.350(0.994)$ & $0.659(0.263)$ & 7.000 & $0.001 *$ & $0.002 *$ \\
\hline Tenericutes & $0.354(0.273)$ & $0.094(0.224)$ & 23.000 & 0.079 & 0.113 \\
\hline Verrucomicrobia & $0.013(0.021)$ & $0.179(0.334)$ & 84.500 & $<0.0005^{*}$ & $<0.0005^{*}$ \\
\hline Other & $0.000(0.002)$ & $0.000(0.004)$ & 49.500 & 0.720 & 0.720 \\
\hline
\end{tabular}

${ }^{*} p<0.05$, Mann-Whitney $\mathrm{U}$ test, Benjamini-Hochberg adjusted $p$ value with $\underline{\underline{\underline{a}}}$ FDR of $0.05 . \mathrm{n}=9$ in $\mathrm{C} 57 \mathrm{BL} / 6$ and $\mathrm{n}=10$ in BTBR group.

Red color indicates an increase; blue color indicates a decrease in the relative abundance of bacterial taxa in BTBR mice.

Figure 2. Table S2. Relative abundance of bacterial phyla BTBR vs C57BL/6 mice. Golubeva, A., et. al. 201 
Figure 2 from the same study shows a table with performed statistics (Mann-Whiteny $U$ test) for the relative abundance of bacterial phyla found in the caecum of the mice groups. These results showed a decrease in the relative abundance of Firmicutes, Proteobacteria, and Cyanobacteria along with an increase of Bacteroidetes and Verrucomicrobia.Results of the overall study showednot only an altered microbiota in BTBR mice composition in comparison to the C57BL/6 but also, deficiency in bile acid and tryptophan metabolism, gastrointestinal dysfunction, and exacerbated social behavior. This was correlated with a reduction in Bifidobacterium and Blautaspecies which are bile metabolizers. Impaired serotonin production correlated with a decreased relative abundance of Firmicutes, specifically Blautia[14]. Taxa previously shown to be associated with ASD include Akkermansia, Bifidobacterium, Blautia, Desulfovibrio, and Biofila [15,16].An increase in gut epithelial cell permeability in the mouse model was also of significant interest as this has been characterized in ASD individuals [17-22]. Knowing this, it can be assumed that gut microbiome therapeutic intervention targeting specific bacterial groups, if applied to a human model, may ameliorate gastrointestinal symptoms, improve microbial-derived intestinal metabolism, better social behavior, and possibly treat ASD.
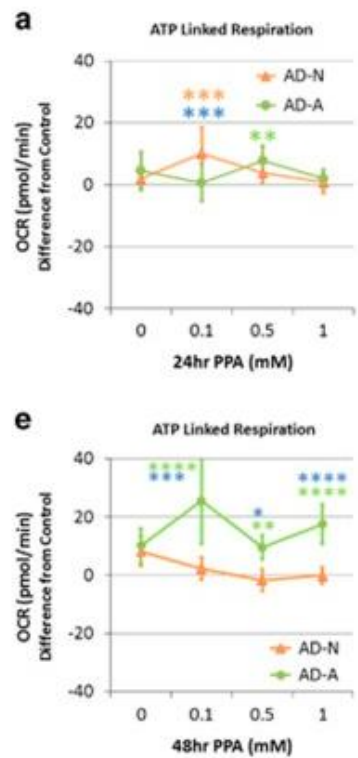
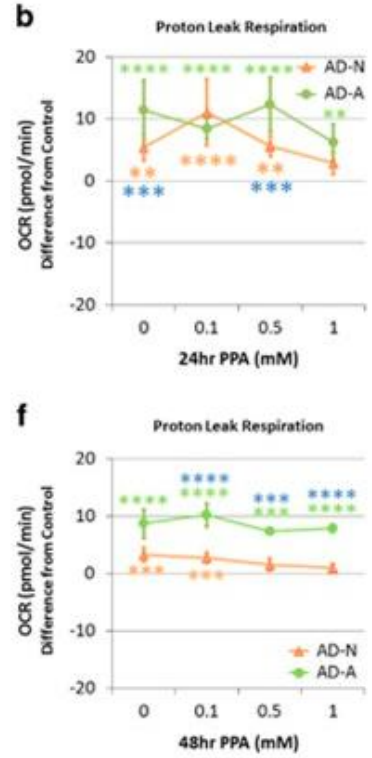
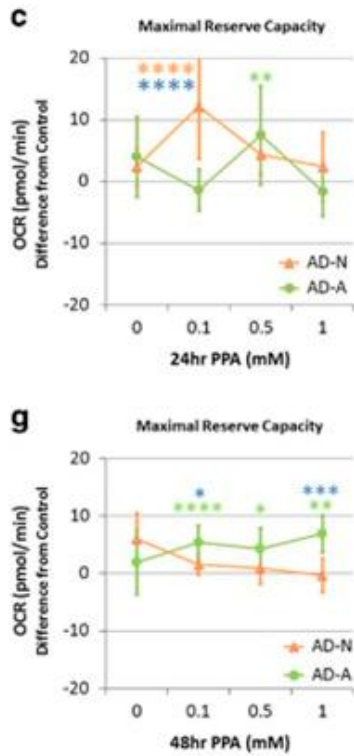
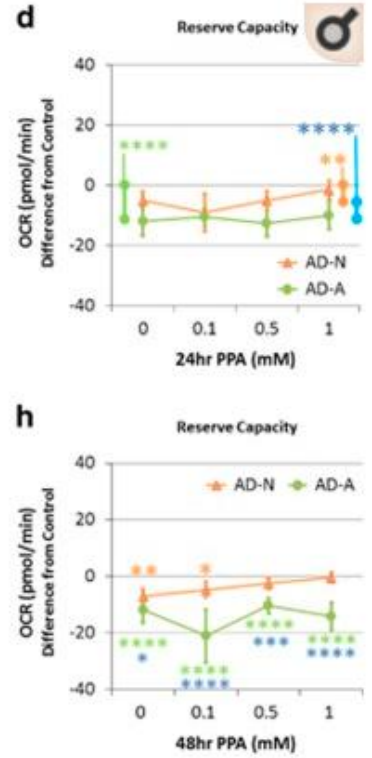

Figure 3. Autistic cell lines: ROS effect on $24 \mathrm{hr}$ and 48hr PPA incubation.Frye, R., et. al. (2016)

Moreover, a human cell line study examined propionic acid (PPA), which is a ubiquitous SCFA that is a product of gut microbiome metabolism, in autism and control cell lines. It has been described that altered HGM and PPA metabolism is correlated with ASD [23-25]. The study aimed to investigate the difference between health and disease effects of PPA by measuring mitochondrial function in ASD and age/gender-matched control lymphoblastoid cell lines following incubation with PPA at different concentrations and durations with and without an in vitro increase in reactive oxygen species [26]. The cell lines were from young white male children donors diagnosed with ASD at a mean age of 8.5 years and controls were obtained from Autism Genetic Resource Exchange, National Institute of Mental Health Center, and Coriell Cell Repository. Seashore assay was used to measure the oxygen consumption rate. Redox challenge, PPA exposure, and 3NP assays were conducted on the cell lines. Analytic approaches used mixed model regression, F-tests, and results reported as P-values.

Figure 3 shows results from reactive oxygen species effects on 24-hour (top) and 48-hour (bottom) PPA incubation. $\mathrm{AD}-\mathrm{A}$ is the autistic cell lines and AD-N is the control cell lines. The data shows that oxidative stress reduces mitochondrial function and reverses the enhancement effect of PPA on mitochondrial function in the AD-A cell lines when compared with AD-N cell lines. This increases when there is a 48-hour PPA incubation with a clearer difference between the two cell lines. The overall outcome of the study, in relation to the HGM, suggests that the cell lines from children with ASD are linked with overproduction of PPA with enteric bacteria that produce PPA being overrepresented. These enteric bacteria are suggested to be Clostridia and Bacteroidetes, which are the main producers of PPA. There have been studies performed that have linked over-representation of Clostridia in children with ASD [2731], ones with regression [32,33], and gastrointestinal symptoms at or before ASD diagnosis or symptom presentations [34].Knowing this, it can be assumed that a form of dietary intervention may reduce the overrepresentation of identified genus of enteric bacterium that are the main producers of PPA and restore mitochondrial function. 

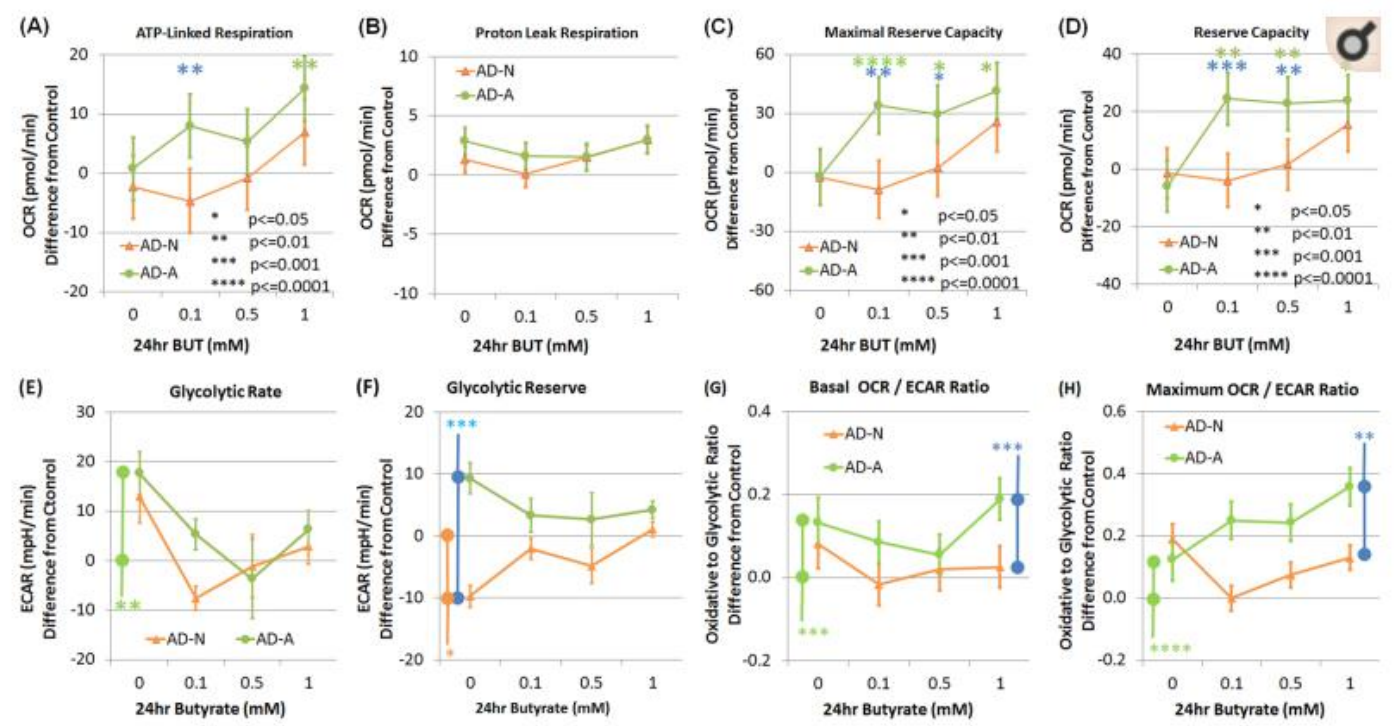

Figure 4. Group differences: the effect of 24h BT exposure on ASD cell lines AD-A and AD-N. Rose et. al. (2018)

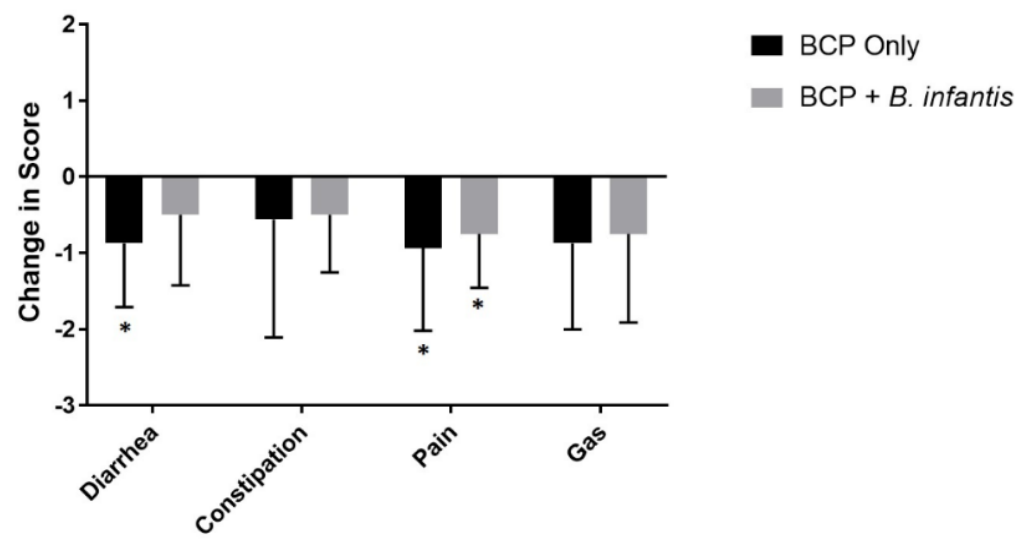

Figure 5. Change in GI symptom frequency with treatment Mean +/- SD change. Significant improvement denoted by asterisk (p<0.05). Sanctuary, M. R., et. al. (2019).

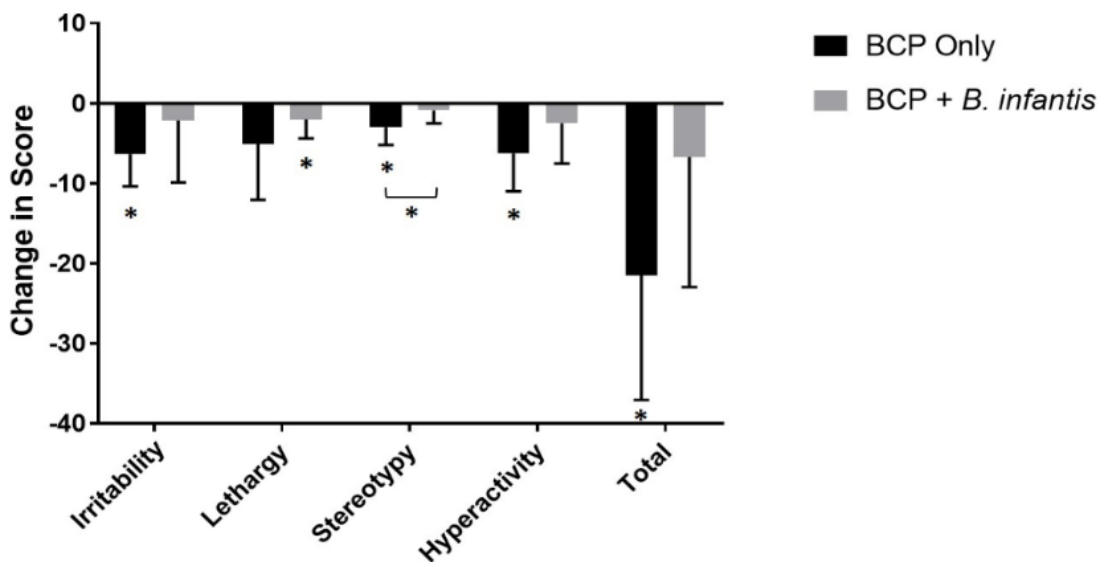

Figure 6. Change in Aberrant Behavior Checklist score with treatment. Mean +/- SD change. Significant improvement denoted by asterisk ( $<<0.05)$ Sanctuary, M. R., et. al. (2019). 
Next, a similar study utilizing similar methods as the study above, examined another ubiquitous SCFA, butyrate (BT). In animal models, it is suggested that BT restores behavior, brain pathophysiology, and pathology [35,36]. BT has also been linked to a more favorable mitochondrial function [37]. Figure 4 shows difference in the effect of 24-hour BT exposure on ASD cell lines. The cell lines have similar annotations as the ones mentioned in the previous study. Differences to note, this had no oxidative stress measure brought upon by ROS and there was not an additional 24-hour observation. Nevertheless, the results showed significant differences between the two groups and were quite intriguing. ATP-linked respiration, proton leak respiration, maximal reserve capacity, and reserve capacity were observed as the study above. Glycolytic rate, glycolytic reserve, basal oxygen consumption rate (OCR) / extracellular acidification rate (ECAR) ratio, and maximum OCR/ECAR ratio was added to these observations. When compared to the PPA study, BT is observed to enhance mitochondrial function in the AD-A cell lines according to the data shown [38]. The overall outcome of the study, in relation to the HGM, is like the PPA study.

An overabundance of these SCFA's by Clostridia species has the possibility of altering mitochondrial function in young individuals with ASD [39].It is to note that enteric bacteria that produce PPA also produce BT. This SCFA is described to aid as mitochondrial fuel which is then used as an energy source for metabolism and suggested through a murine model to rescue deficiency by Butyrivibrio fibrisolvens [40]. Knowing this, it can be assumed that interventional therapeutics of BT may attenuate neurological dysfunctions like ASD through rescuing mitochondrial dysfunction.

\section{HGM, Therapeutics, and ASD}

Having discussed some significant studies regarding HGM, metabolism, and ASD, the puzzle pieces are starting to come closer together. The observed alteration of microbial metabolism, as well as the altered HGM,seems to be a significant contributing factor. Common therapeutic approaches can be assumed to be somewhat dietary in nature. For example, providing the HGM a substrate that is wheat-based promotes enteric bacteria like Clostridia and Bacteroidetes to become more overrepresented and metabolize this to produce PPA [41,42].

A recent review published this year by Virginia Sauraman, Kara Margolis, and Ruth Ann Luna describes some possible microbially mediated therapies that have significant potential for improving quality of life [43]. Dietary interventions such as Gluten, casein, and soy-free diets are described to be commonly studied and/or utilized in ASD populations [44-47] as well as ketogenic diets [48]. Probiotic/prebiotic treatment clinical trials are ongoing, and some are starting to report promising results [49-51]. Antibiotics have been described to alter HGM composition. Knowing this, it can be assumed that this form of therapy may restore favorable HGM composition in ASD and attenuate symptoms. However, clinical trials have been ongoing with some yet to reveal high efficacy [52-54]. Clinical trials using fecal microbiota transplantation have also been performed yielding improvement in both ASD symptoms and gastrointestinal (GI) symptoms [15,55].

Sanctuary et. al. conducted a pilot study aiming to establish supplement tolerability and feasibility for a larger future study with a larger cohort. They assessed tolerability to probiotic (Bifidobacterium infantis) together with a bovine colostrum product (BCP) supplement as a prebiotic in order to evaluate HGM and immune factors in children with ASD and GI co-morbidities [51]. It is to note that tolerability will be measured as children with ASD experience sensory sensitivity which leads to selective diets [56]. This was (study is closed) a double-blind, crossover, phase IIIrandomized clinical trial involving children ages 2-11 with a previous diagnosis of ASD and GI symptoms such as constipation, diarrhea, and irritable bowel (ClinicalTrials.gov identifier: NCT02086110). The study participants (20 total) were recruited locally in Sacramento, California. Nine participants completed the study with eight included in the final analysis. While some mild side effects from prebiotic supplementation included gassiness and weight gain [57], no study participants had to withdraw due to adverse events.

While there was an absence of significant changes in the HGM of the study participants, all study participants experienced a significant reduction in GI symptoms of diarrhea and pain according to the data from the Gastrointestinal History (GIH) survey [58] for BCP only arm (Figure 5). There is also a significant reduction in atypical behavior according to the data from the Aberrant Behavior Checklist $(\mathrm{ABC})$ score in the BCP only armas well (Figure 6). Another interesting find that is of significance is the high initial fecal ethanol and methanol levels that were lowered after treatment.The same statistics were performed as Figures 6 and 7 in relation to fecal alcohol $/ \mathrm{methanol}$ concentrations in the subject stool samples.It has been documented that intestinal yeast overgrowth of Candida or Saccharomyces species causes, not only accumulation of ethanol, but has also been a cause of infection in children with ASD and GI symptoms [59-61]. While the pilot trial had a small sample size, significant data helped support the necessity to continue this study. 
If this were to be continued and analyzed with greater recruitment and a probiotic (B. infantis) only arm,there is a greater possibilitythat it could reveal more about this therapeutic intervention and its efficacy on ASD and GI symptoms.

Furthermore, an increasing amount of research has supported children with ASD having distinct HGM when compared to neurotypical [62-65]. Moreover, murine models reported HGM and metabolite products influencing social behavior through the gut-brain axis [66-68].Consideration of performing FMT as an interventional therapeutic stem from its success as an effective treatment in Clostridium difficile infections which is brought upon by a dysbiotic HGM [69]. Additionally, it has been used to treat other GI/GI associated disorders [70,71].

Kang et. al. conducted an open-label,phase I/II, clinical trial involving 18 children with ASD and GI symptoms (ages 716) and 20 neurotypical without GI symptoms as controls (age and gender-matched).They assessed neurotypical HGM as well as an investigational new treatment called Microbial Transfer Therapy (MTT) in the ASD group for its effectiveness in treating GI and ASD-related symptoms as well as determining effects on

HGM (ClinicalTrials.gov identifier: NCT020504554). Study participants were recruited primarily from Phoenix, Arizona with 3 participants from outside the area. Adverse effects were temporary consisting of mild to moderate hyperactivity and tantrums/aggression [15]. The MTT treatment consisted ofvancomycin, followed by a bowel cleanse, high dose FMT [72], and stomach acid suppressant. Assessments of GI symptoms and ASD symptoms were performed via the Gastrointestinal Rating Scale (GSRS) [73] and ADI-R/PGI-III interview respectively [74,75]. Microbial DNA was extracted from subject feces using a DNA isolation kit and sequenced using MiSeq Illumina platform. Pair-ended sequences targeted 16S rRNA V4 region [76]. Microbiome bioinformatics was analyzed using Quantitative Insights Into Microbial Ecology (QUIIME) [77]. Statistical analysis included Mann-Whitney $U$ test, Wilcoxon signed-rank test, and Spearman's correlation test. $P$ values lower than 0.05 were significant in the data analysis.

This study had an observation of a significant reduction in GI symptoms. GSRS scores were improved for symptoms such as abdominal pain, indigestion, diarrhea, and constipation following treatment, even 8 weeks after. This was proved via a two-tailed Wilcoxon signed-rank test witha p-value of $<0.001$. ASD symptoms also improved following treatment and no regression 8 weeks after as well. This was also proved via the same statistical analysis used for the GSRS scores with a similar p-value. Gut bacterial composition using the phylogenetic diversity index [78] was significantly less diverse in the ASD group when compared to the neurotypical group at baseline. This was observed using a one-tailed Mann-Whitney $U$ test with a p-value of 0.027. At the end of treatment, significant bacterial diversity increased in the ASD group and remained 8 weeks after treatment (two-tailed Wilcoxon signed-rank, $\mathrm{p}<0.05$ and $\mathrm{p}=$ 0.001 )was discontinued with almost identicalrichness compared to the control group after the $18^{\text {th }}$ week (two-tailed Mann-Whitney $U$ test, $\mathrm{p}=0.78$ ). Significant increase in relative abundances of bacterial genera following MTT treatment were Bifidobacterium, Prevotella, and Desulfovibrio with (two-tailed Mann-Whiteny $U$ test $\mathrm{p}<0.05$ ). Bifidobacterium underrepresentation has been previously reported in ASD individuals[79,80].

Furthermore, this study has been intriguing in its observation of MTT treatment restoring HGM composition in ASD individuals to that of neurotypical. It also further supports that an association exists between HGM and GI/ASD symptoms. However, the study had its limitations as it was not placebo-controlled, blinded, or randomized. A follow up to this study would most likely have to take into consideration vancomycin only control arm to determine its effects and compare with the rest of the arms. The same goes for bowel cleanse, FMT, and stomach acid suppressant. Larger sample sizes would also be considered and possible geographic restrictions.

Moreover, long-termeffects of treatment were evaluated in the 18 ASD participants 2 years after the discontinuation of MTT [55]. Significant improvements in GI and ASD symptoms, as well as HGM community diversity and relative abundance of Bifidobacteria, Desulfovibrio, and Prevotella, were observed in the 2 year follow up when compared with baseline. An increase in Prevotella is of significance as it has been observed to be in low abundance in ASD when compared with neurotypical [81]. Statistical methods that were used which lead to these conclusions were the same as the initial trial, two-tailed Mann-Whitney $U$ test and two-tailed Wilcoxon signed-rank. These observations strongly consider continuing the study with the previously discussed alteration in study design. 


\section{Concluding Remarks}

The HGM and its metabolic products have been observed in these investigations to play a significant role in ASD. Whether directly through the alteration of gut microbial composition or indirectly via gut microbial metabolism products. Diet is seen to be a major factor in contributing to not only exacerbated GI symptoms but also in worsening social behavior. Given the more restricted diets of individuals with ASDcompared with neurotypical, the studies support differences in HGM, as well as microbial metabolic products, produced such as BT and PPA. Follow up to these studies may benefit from determining levels of different SCFA's like acetate. The dietary interventions discussed utilizing prebiotics and/or probiotics has some promise.Even more so is the more interventional FMT treatment or MTT. Follow up to these studies may benefit from investigations into the efficacy of using prebiotic vs. probiotic vs. FMT or some other study design that is similar in order to assess HGM compositions. The clinical trials observed in these studies while having significant observations, suffered from a small sample size. Alternative approaches in recruitment should be considered in order to recruit as many ASD and neurotypical individuals as possible. Geographical environment, antibiotic medications, and even extraintestinal symptoms maybe another variable to take into consideration when assessing a clinical trial of this nature. Overall, studies have been continuing and have been seeing promise as well with little to no adverse events. It is just a matter a time before the puzzle pieces finally fit and have a solution to this neurological enigma.

\section{References}

Christensen, D. L., et. al. (2018). Prevalence and characteristics of autism spectrum disorder among children aged 8 years - autism and developmental disabilities monitoring network, 11 sites, United States, 2012. MMWR Surveillance Summaries, 65(13), 1.

Berding, K., and Donovan, S. (2016). Microbiome and nutrition in autism spectrum disorder: Current knowledge and research needs. Nutrition Reviews, 74(12).

Cox, L., and Weiner, H. (2018). Microbiota Signaling Pathways that Influence Neurologic Disease. Neurotherapeutics : the journal of the American Society for Experimental NeuroTherapeutics, 15(1), 135-145.

Adams, J. B., and Holloway, C. (2004). Pilot study of a moderate dose multivitamin/mineral supplement for children with autistic spectrum disorder. Journal of Alternative \& Complementary Medicine, 10(6), 1033-1039.

Tengeler, A. C., et. al. (2018). Relationship between diet, the gut microbiota, and brain function. Nutrition reviews, 76(8), 603-617.

Wikoff, W. R., et. al. (2009). Metabolomics analysis reveals large effects of gut microflora on mammalian blood metabolites. Proceedings of the national academy of sciences, 106(10), 3698-3703.

Agus, A., et. al. (2018). Gut Microbiota Regulation of Tryptophan Metabolism in Health and Disease. Cell host \& microbe, 23(6), 716-724.

Yano, J. M., et. al. (2015). Indigenous bacteria from the gut microbiota regulate host serotonin biosynthesis. Cell, 161(2), 264-276.

Hoshino, Y., et. al. (1986). Plasma free tryptophan concentration in autistic children. Brain and Development, 8(4), 424-427.

Kałuzna-Czaplinska, J., et. al. (2010). Determination of tryptophan in urine of autistic and healthy children by gas chromatography/mass spectrometry. Medical Science Monitor, 16(10), CR488-CR492.

Adams, J. B., et. al. (2011). Nutritional and metabolic status of children with autism vs. neurotypical children, and the association with autism severity. Nutrition \& metabolism, 8(1), 34. https://doi.org/10.1186/1743-7075-8-34

Naushad, S. M., et. al. (2013). Autistic children exhibit distinct plasma amino acid profile.

Kałużna-Czaplińska, J., et. al. (2019). How important is tryptophan in human health? Critical Reviews in Food Science and Nutrition, 59(1), 72-88.

Golubeva, A., et. al. (2017). Microbiota-related Changes in Bile Acid \& Tryptophan Metabolism are Associated with Gastrointestinal Dysfunction in a Mouse Model of Autism. EBioMedicine, 24.

Kang, D. W., et. al. (2017). Microbiota Transfer Therapy alters gut ecosystem and improves gastrointestinal and autism symptoms: an open-label study. Microbiome, 5(1), 10.

Vuong, H. E., \& Hsiao, E. Y. (2017). Emerging roles for the gut microbiome in autism spectrum disorder. Biological psychiatry, 81(5), 411-423.

De Angelis, M., et. al. (2013). Fecal microbiota and metabolome of children with autism and pervasive developmental disorder not otherwise specified. PloS one, 8(10).

d'Eufemia, P., et. al. (1996). Abnormal intestinal permeability in children with autism. Acta paediatrica, 85(9), 10761079. 
Kang, D. W., et. al. (2013). Reduced incidence of Prevotella and other fermenters in intestinal microflora of autistic children. PloS one, 8(7).

Luna, R. A., et. al. (2017). Distinct microbiome-neuroimmune signatures correlate with functional abdominal pain in children with autism spectrum disorder. Cellular and molecular gastroenterology and hepatology, 3(2), 218230.

McElhanon, B. O., et. al. (2014). Gastrointestinal symptoms in autism spectrum disorder: a meta-analysis. Pediatrics, $133(5), 872-883$.

Tomova, A., et. al. (2015). Gastrointestinal microbiota in children with autism in Slovakia. Physiology \& behavior, $138,179-187$.

MacFabe, D. F. (2015). Enteric short-chain fatty acids: microbial messengers of metabolism, mitochondria, and mind: implications in autism spectrum disorders. Microbial ecology in health and disease, 26(1), 28177.

Frye, R. E., et. al. (2015). Gastrointestinal dysfunction in autism spectrum disorder: the role of the mitochondria and the enteric microbiome. Microbial ecology in health and disease, 26(1), 27458.

Frye, R. E., et. al. (2015). Approaches to studying and manipulating the enteric microbiome to improve autism symptoms. Microbial ecology in health and disease, 26(1), 26878.

Frye, R., et. al. (2016). Modulation of mitochondrial function by the microbiome metabolite propionic acid in autism and control cell lines. Translational psychiatry, 6(10), e927.

De Angelis, M., et. al. (2013). Fecal microbiota and metabolome of children with autism and pervasive developmental disorder not otherwise specified. PloS one, 8(10).

Keşli, R., et. al. (2014). Investigation of the relation between anaerobic bacteria genus clostridium and late-onset autism etiology in children. Journal of Immunoassay and Immunochemistry, 35(1), 101-109.

Finegold, S. M. (2008). Therapy and epidemiology of autism-clostridial spores as key elements. Medical hypotheses, $70(3), 508-511$.

Parracho, H. M., et. al. (2005). Differences between the gut microflora of children with autistic spectrum disorders and that of healthy children. Journal of medical microbiology, 54(10), 987-991.

Song, Y., et. al. (2004). Real-time PCR quantitation of clostridia in feces of autistic children. Appl. Environ. Microbiol., 70(11), 6459-6465.

Finegold, S. M., et. al (2012). Microbiology of regressive autism. Anaerobe, 18(2), 260-262.

Finegold, S. M., et. al. (2002). Gastrointestinal microflora studies in late-onset autism. Clinical Infectious Diseases, 35(Supplement_1), S6-S16.

Williams, B. L., et. al. (2011). Impaired carbohydrate digestion and transport and mucosal dysbiosis in the intestines of children with autism and gastrointestinal disturbances. PloS one, 6(9).

Takuma, K., et. al. (2014). Chronic treatment with valproic acid or sodium butyrate attenuates novel object recognition deficits and hippocampal dendritic spine loss in a mouse model of autism. Pharmacology biochemistry and behavior, 126, 43-49.

Kratsman, N., et. al. (2016). Sodium butyrate attenuates social behavior deficits and modifies the transcription of inhibitory/excitatory genes in the frontal cortex of an autism model. Neuropharmacology, 102, 136-145.

Hong, J., et. al. (2016). Butyrate alleviates high fat diet-induced obesity through activation of adiponectin-mediated pathway and stimulation of mitochondrial function in the skeletal muscle of mice. Oncotarget, 7(35), 56071.

Rose, S., et. al. (2018). Butyrate enhances mitochondrial function during oxidative stress in cell lines from boys with autism. Translational psychiatry, 8(1), 1-17.

Frye, R. E., et. al. (2013). Unique acyl-carnitine profiles are potential biomarkers for acquired mitochondrial disease in autism spectrum disorder. Translational psychiatry, 3(1), e220-e220.

Donohoe, D. R., et. al. (2011). The microbiome and butyrate regulate energy metabolism and autophagy in the mammalian colon. Cell metabolism, 13(5), 517-526.

Haskå, L., et. al. (2011). A water-soluble fraction from a by-product of wheat increases the formation of propionic acid in rats compared with diets based on other by-product fractions and oligofructose. Food \& nutrition research, $55(1), 6397$.

Haskå, L., et. al. (2011). The effect of dietary fiber from wheat processing streams on the formation of carboxylic acids and microbiota in the hindgut of rats. Journal of agricultural and food chemistry, 59(7), 3406-3413.

Saurman, V., et. al. (2020). Autism Spectrum Disorder as a Brain-Gut-Microbiome Axis Disorder. Digestive Diseases and Sciences, 1-11.

Hyman, S. L., et. al. (2016). The gluten-free/casein-free diet: a double-blind challenge trial in children with autism. Journal of autism and developmental disorders, 46(1), 205-220.

Navarro, F., et. al. (2015). Are 'leaky gut' and behavior associated with gluten and dairy containing diet in children with autism spectrum disorders?. Nutritional neuroscience, 18(4), 177-185. 
Ghalichi, F., et. al. (2016). Effect of gluten free diet on gastrointestinal and behavioral indices for children with autism spectrum disorders: a randomized clinical trial. World Journal of Pediatrics, 12(4), 436-442.

Adams, J. B., et. al. (2018). Comprehensive nutritional and dietary intervention for autism spectrum disorder-a randomized, controlled 12-month trial. Nutrients, 10(3), 369.

El-Rashidy, O., et. al. (2017). Ketogenic diet versus gluten free casein free diet in autistic children: a case-control study. Metabolic brain disease, 32(6), 1935-1941.

Liang, D., et. al. (2019). Efficacy of different probiotic protocols in irritable bowel syndrome: A network metaanalysis. Medicine, 98(27).

Arnold, L. E., et. al. (2019). Probiotics for Gastrointestinal Symptoms and Quality of Life in Autism: A PlaceboControlled Pilot Trial. Journal of child and adolescent psychopharmacology, 29(9), 659-669.

Sanctuary, M. R., et. al. (2019). Pilot study of probiotic/colostrum supplementation on gut function in children with autism and gastrointestinal symptoms. PloS one, 14(1).

Sandler, R. H., et. al. (2000). Short-term benefit from oral vancomycin treatment of regressive-onset autism. Journal of child neurology, 15(7), 429-435.

Ghaleiha, A., et. al. (2016). Minocycline as adjunctive treatment to risperidone in children with autistic disorder: a randomized, double-blind placebo-controlled trial. Journal of child and adolescent psychopharmacology, 26(9), 784-791.

Minshawi, N. F., et. al. (2016). A randomized, placebo-controlled trial of D-cycloserine for the enhancement of social skills training in autism spectrum disorders. Molecular autism, 7(1), 2.

Kang, D. W., et. al. (2019). Long-term benefit of Microbiota Transfer Therapy on autism symptoms and gut microbiota. Scientific reports, 9(1), 5821.

Cermak, S. A., et. al. (2010). Food selectivity and sensory sensitivity in children with autism spectrum disorders. Journal of the American Dietetic Association, 110(2), 238-246.

Goetze, O., et. al. (2008). Effect of a prebiotic mixture on intestinal comfort and general wellbeing in health. British journal of nutrition, 100(5), 1077-1085.

Hertz-Picciotto, I., et. al. (2006). The CHARGE study: an epidemiologic investigation of genetic and environmental factors contributing to autism. Environmental health perspectives, 114(7), 1119-1125.

Hunnisett, A., et. al. (1990). Gut fermentation (or the 'Auto-brewery') Syndrome: A new clinical test with initial observations and discussion of clinical and biochemical implications. Journal of Nutritional Medicine, 1(1), 33-38.

Cordell, B., and McCarthy, J. (2013). A case study of gut fermentation syndrome (auto-brewery) with Saccharomyces cerevisiae as the causative organism. International Journal of Clinical Medicine, 2013.

Iovene, M. R., et. al. (2017). Intestinal dysbiosis and yeast isolation in stool of subjects with autism spectrum disorders. Mycopathologia, 182(3-4), 349-363.

Finegold, S. M., et. al. (2010). Pyrosequencing study of fecal microflora of autistic and control children. Anaerobe, 16(4), 444-453.

Williams, B. L., et. al. (2012). Application of novel PCR-based methods for detection, quantitation, and phylogenetic characterization of Sutterella species in intestinal biopsy samples from children with autism and gastrointestinal disturbances. MBio, 3(1), e00261-11.

Gondalia, S. V., et. al. (2012). Molecular characterisation of gastrointestinal microbiota of children with autism (with and without gastrointestinal dysfunction) and their neurotypical siblings. Autism Research, 5(6), 419-427.

Son, J. S., et. al. (2015). Comparison of fecal microbiota in children with autism spectrum disorders and neurotypical siblings in the simons simplex collection. PloS one, 10(10).

Hsiao, E. Y., et. al. (2013). Microbiota modulate behavioral and physiological abnormalities associated with neurodevelopmental disorders. Cell, 155(7), 1451-1463.

Kim, S., et. al. (2017). Maternal gut bacteria promote neurodevelopmental abnormalities in mouse offspring. Nature, 549(7673), 528-532.

Buffington, S. A., et. al. (2016). Microbial reconstitution reverses maternal diet-induced social and synaptic deficits in offspring. Cell, 165(7), 1762-1775.

Bagdasarian, N., et. al. (2015). Diagnosis and treatment of Clostridium difficile in adults: a systematic review. Jama, 313(4), 398-408.

Moayyedi, P., et. al. (2015). Fecal microbiota transplantation induces remission in patients with active ulcerative colitis in a randomized controlled trial. Gastroenterology, 149(1), 102-109.

$\mathrm{Xu}$, M. Q., et. al. (2015). Fecal microbiota transplantation broadening its application beyond intestinal disorders. World Journal of Gastroenterology: WJG, 21(1), 102. 
Hamilton, M. J., et. al. (2012). Standardized frozen preparation for transplantation of fecal microbiota for recurrentClostridium difficileinfection. American Journal of Gastroenterology, 107(5), 761-767.

Revicki, D. A., et. al. (1997). Reliability and validity of the Gastrointestinal Symptom Rating Scale in patients with gastroesophageal reflux disease. Quality of life research, 7(1), 75-83.

Lord, C., et. al. (1994). Autism Diagnostic Interview-Revised: a revised version of a diagnostic interview for caregivers of individuals with possible pervasive developmental disorders. Journal of autism and developmental disorders, 24(5), 659-685.

Adams, J. B., et. al. (2011). Effect of a vitamin/mineral supplement on children and adults with autism. BMC pediatrics, 11(1), 111.

Caporaso, J. G., et. al. (2012). Ultra-high-throughput microbial community analysis on the Illumina HiSeq and MiSeq platforms. The ISME journal, 6(8), 1621-1624.

Caporaso, J. G., et. al. (2010). QIIME allows analysis of high-throughput community sequencing data. Nature methods, $7(5), 335$.

Faith, D. P. (1992). Conservation evaluation and phylogenetic diversity. Biological conservation, 61(1), 1-10.

Adams, J. B., et. al. (2011). Gastrointestinal flora and gastrointestinal status in children with autism-comparisons to typical children and correlation with autism severity. BMC gastroenterology, 11(1), 22.

Wang, L., et. al. (2011). Low relative abundances of the mucolytic bacterium Akkermansia muciniphila and Bifidobacterium spp. in feces of children with autism. Appl. Environ. Microbiol., 77(18), 6718-6721.

Kang, D. W., et. al. (2018). Differences in fecal microbial metabolites and microbiota of children with autism spectrum disorders. Anaerobe, 49, 121-131. 